Of the 2,803 papers found, 31 were included. Our preliminary findings show that HCWs are at increased risk for stress, burnout and depression during the COVID-19 pandemic. These negative outcomes are triggered by individual-level factors such as gender, family status and lack of social support; organizational-level factors such as high workload and access to PPE; and systemic-level factors such as prevalence of COVID-19, rapidly changing public health guidelines and a lack of recognition at work. There is a limited amount of evidence on effective interventions that prevent anxiety, stress, burnout and depression during a pandemic. Preliminary findings of causes of increased stress and mental health issues suggest possible strategies healthcare organizations can use to address modifiable factors such as ongoing training to increase confidence in caring for COVID-19 patients, clear infection control guidelines and sufficient PPE and optimization of working conditions for HCWs.

\section{Women's health}

\section{OPTIMISING THE CLINICAL DVT PATHWAY IN THE MATERNITY ASSESSMENT UNIT}

${ }^{1}$ Skukura Murray, 'Elizabeth Leftley, ${ }^{2}$ Jess McMicking, ${ }^{2}$ Adam D Jakes, ${ }^{2}$ Anne Cobell, ${ }^{2}$ Manju Chandiraman. 'King's College London, GKT Medical School, UK; ' ${ }^{2}$ Guy's and St Thomas' NHS Foundation Trust

10.1136/leader-2020-FMLM.180

Aim The risk of a deep vein thrombosis (DVT) increases in pregnancy, occurring in 1-2 in 1000 women. The aim of our quality improvement project was to streamline and increase the efficiency of the assessment and treatment of Deep Vein Thrombosis in antenatal and postnatal (up to 6 weeks) women, presenting to the Maternity Assessment Unit (MAU) at St Thomas' Hospital.

Method The maternity data collection system (BadgerNet, Clevermed Ltd) was used to conduct a retrospective analysis of women presenting to the MAU with symptoms suggestive of a DVT between July 2018 and March 2019. Staff were educated on the updates to Trust guidelines on diagnosis and management of DVT in pregnancy. The need for a doctor to review a patient prior to same day ultrasound Doppler scan was eliminated and nurses were given the ability to request an ultrasound Doppler if a woman met agreed criteria. A re-audit was completed between August 2019 and March 2020.

Results The elimination of a doctor review prior to same day ultrasound Doppler scans, reduced waiting time for patients and allowed doctors to assess with the complete clinical information. There has been a reduction in unwarranted clinical variance in the assessment and treatment of DVTs, with 78\% compliance with the protocol (100\% for same day scans, $62 \%$ for next day scans). 3 patients did not receive Low Molecular Weight Heparin (LMWH) according to the new protocol, one of which declined treatment.

Conclusion Clear, concise guidelines for staff reduces unwarranted clinical variation and ensures safer management of patients. The extension of the midwife's role within the MAU provided midwives with more clinical autonomy and reduced delays in assessment and treatment. Assessment by a doctor prior to the ultrasound request did not contribute to improved patient care.

\section{Leading innovation and improvement}

\section{HARNESSING STAFF VALUES TO CATALYSE WORKPLACE CHANGE}

Nadia Audhali, Anna Moore, Tim Martin, Charlotte Munro, Florence Wedmore. Barts Health Trust, London, UK

\subsection{6/leader-2020-FMLM.181}

Green@Bart's is a 60 strong multidisciplinary staff special interest group at one the UK's largest Acute Foundation Trusts. All working on a voluntary basis and united by a passion for sustainability and climate action.

Despite the WHO stating climate change as the most significant Public Health issue of our generation, reducing carbon emissions from healthcare remains a huge challenge, and ever-bolder legal targets eg 'Net Zero' emissions by 2050, require innovative thinking and support at every level to succeed.

Guided by our passion for addressing these issues Green@Barts had the courage to approach senior leadership through Board meeting thereby winning Chief Executive support and valuable networking opportunities to enable our work.

Within one year of its inception Green@Barts are now invited by Trust leaders for our input. We are currently consulting on post-Covid transformational work for Trust-wide outpatients and emergency department. We have shared best practice with staff at other Trusts, supporting them to set up their own sustainability staff group, and there are plans for a mini-conference. We see the necessity of winning hearts and minds and education and awareness raising for staff and patients run throughout our work.

Other work ranges from providing scrutiny and comments on the Trust's key sustainability and emission reduction document- the SDMP aka The Green Plan, as well as the Architect Tender document for Whipps Cross redevelopment, thereby securing an aspiration for the UK's first 'Carbon Net Zero' hospital.

Secondary beneficial outcomes include promoting staff engagement and belonging, both key for promoting resilience and reducing burnout.

Connecting through shared values can powerfully support a change agenda, and this can be driven by highly motivated staff with the courage to speak up. This bottom up approach complements the top down work from Trust leadership, vital to achieving maximum results.

\section{Leading across systems and organisations}

\section{THE IMPACT OF COVID-19 ON COMMUNICATION IN THEATRES}

Danielle Banfield, Ashish Patel, Alexandra Humphreys, Joanne Bennett. Gloucestershire Hospitals NHS Foundation Trust

\subsection{6/leader-2020-FMLM.182}

Gloucestershire Hospitals NHS Foundation trust comprises sites in Cheltenham and Gloucester (GRH). As part of the COVID-19 response, emergency services were temporarily 
reconfigured to GRH. This was rapidly implemented, to maximise patient safety whilst managing high patient volumes and potentially high levels of staff sickness.

In theatres, communication is critical to safe working. Much has been done in our Trust to improve theatre briefings. We aimed to assess how communication in theatres was impacted by changes made to working patterns during COVID-19. A SurveyMonkeyTM collected demographics, and Likert scale responses assessing team working, communication, briefings, and staff well-being. The survey was distributed to all surgical specialties, anaesthetic, and theatre staff. A total of 100 responses were collected over 5 weeks.

The survey confirmed that COVID-19 had a significant impact on working structures. $60 \%$ of respondents worked in different teams, and $44 \%$ felt outside of their comfort zone. $72 \%$ found communication more difficult, with $92 \%$ attributing this to PPE. The majority (72\%) felt briefings covered specific C-19 related issues and they could raise concerns $(77 \%)$. However, only $26 \%$ of respondents felt this was true for emergency briefings. This is the focus of our initial change.

We plan to improve briefings whilst re-engaging those stakeholders who were less involved during the early phase of the initial pandemic. Our high volume of emergency work means that we can implement rapid PDSA cycles following focused discussion groups. Some simple changes have already been made, including introduction of a dedicated pager for the operating general surgeon, and allocation of a CEPOD co-ordinator to attend morning handover. We plan to repeat the survey in 6 weeks. We will also conduct interviews with staff which, in addition to evaluating service improvement, will give vital information to guide ongoing refinements.

\section{Leading across systems and organisations}

\section{DEVELOPMENT OF A COVID-19 PCR TESTING PATHWAY FOR KEY WORKERS AND CARE HOMES}

${ }^{1}$ Mary C Self, ${ }^{2}$ Neda Mehrpooya. ${ }^{1}$ Mental Health Rehabilitation Services, Cwm Taf Morgannwg University Health Board, Wales; ${ }^{2}$ Forensic Mental Health Services, Swansea Bay University Health Board, Wales

10.1136/leader-2020-FMLM.183

The Doctors Laboratory Group (TDL) has provided pathology testing for over thirty years, twenty of those as NHS partners. TDL runs a national network of UKAS accredited hub and spoke laboratories. Throughout the Covid-19 pandemic TDL Group, has supported:

- Pillar 1 NHS PCR Covid-19 tests

- Francis Crick Institute and UCL Pillar 2

- Pillar 3 antibody testing

- Pillar 4 research projects

- MedCity surge capacity

- London Nightingale Hospital

- Testing for professional sport, media and commerce

In late April Brent and Harrow CCGs required a Covid19 PCR testing pathway for symptomatic key workers and suspected care home outbreaks. Brent and Harrow were named the top two London Boroughs worst affected by
Covid-19 deaths, with Brent topping the UK data for Covid19 deaths.

Covid- 19 Hubs and Rapid Response Teams were formed with logistics, IT connectivity and training for electronic requesting and reporting all signed-off within 6 days. Pivotal to this success were Lead Contacts who overcame challenges from testing in non-standard locations, coordinating Multi-Disciplinary Teams (MDT) across organisations, new social distancing and lockdown rules, international shortages of consumables, identifying key worker and patient requests for rapid notification of results and NHSE reporting.

Fundamental to the project's success were strong Leads for each organisation who understood their team skills so tasks were appropriately delegated. Leads worked together to maintain momentum, set actions and deadlines, provide rapid resolution to queries or potential stumbling blocks and praise teams on work achieved, promoting ongoing engagement.

Covid-19 testing is key to preventing virus spread and the protection of patients. The hub-spoke model underpinning The Doctors Laboratory Group has proven to be highly adaptable and resilient to exponential, rapid growth. This project's success demonstrates that with the right leadership big changes can happen over a few days.

\section{Education}

\section{ASSESSMENT OF VIRTUAL TEACHING FOR OBSTETRIC AND GYNAECOLOGY POSTGRADUATE TRAINEES IN NORTHERN IRELAND}

${ }^{1} \mathrm{G}$ Ferguson, ${ }^{2} \mathrm{~J}$ McManus, ${ }^{3} \mathrm{R}$ Henry, ${ }^{4} \mathrm{~N}$ Haughey. ${ }^{1}$ The Royal Victoria Hospital, Belfast, UK; ${ }^{2}$ The Royal Victoria Hospital, Belfast, UK; ${ }^{3}$ Craigavon Area Hospital, Craigavon, UK; ${ }^{4}$ Antrim Area Hospital, Antrim, UK

\subsection{6/leader-2020-FMLM. 184}

Background Obstetrics and Gynaecology trainees in Northern Ireland attend bi-monthly postgraduate continued medical education (CME). COVID-19 restrictions meant these teaching sessions could not occur. Collaborating with senior trainees we developed a virtual postgraduate teaching series via ZOOM, delivered over two months focusing on key areas of the new Royal College of Obstetricians and Gynaecologists (RCOG) curriculum.

Aims To assess whether virtual teaching is an effective platform to deliver CME for obstetrics and gynaecology trainees in Northern Ireland.

Methods Survey monkey sent to participants, non-participants and consultants exploring experiences and attitudes towards this teaching series.

Results 33 trainees completed the participant survey, with the majority attending 1-2 sessions. Over 50\% were first time users of video conference based teaching. $66 \%$ felt confident using video-conferencing for learning. 27 participants agreed the pre session reading enhanced learning. All participants agreed CME should be mapped to the RCOG curriculum. $87 \%$ of participants agreed that video-conferencing from a location of choosing improves accessibility.

15 trainees completed the non-participant survey, with 93\% stating work commitments as the non-attendance reason. Interestingly 2 trainees were unable to work ZOOM. 73\% of non- 